\title{
Diltiazem treatment for the management of ischaemia in patients with poor left ventricular function: safety of long term administration
}

\author{
MICHAEL J ZEMA, * STEVEN PERLMUTTER, $\dagger$ SETH MANKES, $\dagger$ \\ CHRISTOPHER NIKITOPOULOS *
}

From the ${ }^{\star}$ Department of Medicine, Division of Cardiology; and the $†$ Department of Radiology, Brookhaven Memorial Hospital Medical Center, Patchogue, New York, USA

SUMMARY The tendency of oral diltiazem (a calcium entry blocking agent and a negative inotrope) to induce or exacerbate congestive heart failure when used for the long term management of myocardial ischaemia in patients with poor left ventricular function has not been investigated before. Twenty two patients (aged 42-73 years) with pretreatment left ventricular ejection fraction ranging from 0.11 to 0.39 were given open label oral dialtiazem $(120-360 \mathrm{mg} / 24 \mathrm{~h}$ (mean $254 \mathrm{mg}$ ) for two weeks to 16 months (mean 7.5 months, median 6.2 months). There was a weight change of $>3 \mathrm{lb}(1.35 \mathrm{~kg})$ in nine patients-five gained weight and four lost it. Diltiazem treatment did not alter the mean (SD) cardiothoracic ratio on chest $x$ ray $(0.47(0.06)$ before $v s 0.48(0.05)$ after $)$ or the left ventricular ejection fraction at rest $(0.28(0.09)$ before $v s 0.26(0.08)$ after $)$. Diltiazem was discontinued in one patient because of symptoms indicative of worsening congestive heart failure. No patient required admission to hospital for treatment of symptoms resulting from further left ventricular decompensation. Diltiazem was discontinued in six other patients for other reasons.

Long term administration of oral diltiazem was not regularly associated with a deterioration in clinical, radiographic, or radionuclide ventriculographic estimates of left ventricular function, even in patients with poor baseline left ventricular systolic performance.

Agents that block calcium entry are potentially capable of altering left ventricular function by three basic mechanisms: (a) direct peripheral arterial dilatation or left ventricular afterload reduction; $(b)$ reflex augmentation of sympathetic nervous activity; and $(c)$ direct myocardial inotropic depression. ${ }^{12}$ The first two of these effects would tend to augment performance and counterbalance the direct negative inotropic effect. The net cardiocirculatory response to such agents may differ depending upon the calcium entry blocking agent used, ${ }^{34}$ the dose, the use of concomitant cardioactive drugs, ${ }^{5-7}$ and the haemodynamic state of the patient. ${ }^{8}$ There is concern about the potential myocardial depressant effects of these

Requests for reprints to Dr Michael J Zema, Department of Medicine, Division of Cardiology, Brookhaven Memorial Hospital Medical Center, 101 Hospital Road, Patchogue, New York 11772, USA.

Accepted for publication 1 July 1987 agents when they are used in patients with abnormally depressed left ventricular performance.

There are few data on the safety of diltiazem treatment in patients with severe left ventricular dysfunction. The few data available were obtained from patients given short term intravenous diltiazem ${ }^{910}$ or from patients treated orally for less than 24 hours. ${ }^{10}$ The safety of diltiazem is of more than academic interest. Adequate control of angina pectoris with nitrate preparations alone is often not possible. Coronary artery vein bypass surgery, moreover, is associated with high perioperative morbidity and mortality in this group of patients. ${ }^{11}$ Several studies have suggested preservation of ischaemic myocardium with diminished infarct size in animals pretreated with diltiazem before the induction of experimental myocardial infarction. ${ }^{12}$ Such studies suggest that diltiazem may have a role in the management of the patient with severely depressed left ventricular function after myocardial infarction in 
whom the prophylactic use of $\beta$ adrenergic blocking agents may precipitate congestive heart failure. ${ }^{13-15}$ Accordingly, we designed the present prospective open label investigation to assess the effects of long term oral diltiazem on left ventricular performance in a group of patients with poor baseline left ventricular function who were treated for myocardial ischaemia.

\section{Patients and methods}

\section{PATIENTS}

Twenty two patients (21 men and one woman, mean age 60 years, range $42-73$ ) with mean left ventricular ejection fraction at rest of 0.28 (range $0 \cdot 11-0.39$ ) were treated with oral diltiazem. All patients had had a myocardial infarction $>$ two weeks ago and 11 had had multiple previous infarctions. Indications for diltiazem included stable or unstable clinical angina pectoris in 14 subjects, electrocardiographic evidence of ischaemia in the absence of chest pain on exercise (silent ischaemia) in five, and coronary artery spasm refractory to nitrate treatment in one. Two patients received the drug prophylactically from two weeks after myocardial infarction. No patient was treated with diltiazem for the sole purpose of controlling the symptoms of left heart failure.

Before treatment with oral diltiazem for ischaemia was started the symptoms and signs of left ventricular dysfunction had been treated with oral or topical nitrates in $15(68 \%)$ patients, digitalis glycoside in 11 $(50 \%)$, diuretics in $10(45 \%)$, converting enzyme inhibitor or direct arteriolar vasodilators in three $(14 \%)$, and $\alpha$ adrenergic blockade in one (5\%). Compensation on these regimens was good. Six $(27 \%)$ had symptoms of congestive heart failure including dyspnoea upon exertion, four $(18 \%)$ had orthopnoea, and two $(9 \%)$ had paroxysmal nocturnal dyspnoea.

The following additional cardiac medications were continued: antiarrhythmic treatment in five $(23 \%)$, warfarin anticoagulation in three $(14 \%)$, and antiplatelet treatment in three $(14 \%)$. Many patients were taking more than one medication concomitantly. No patient was being treated with a $\beta$ adrenergic blocking agent.

\section{CHEST X RAY PROCEDURE}

Routine posteroanterior chest radiographs were obtained in all patients before treatment with oral diltiazem and again a mean of 20 weeks (range 3-55) later in 19. The films were independently interpreted by two radiologists blinded to the patients' clinical state. The chest $x$ rays were graded for congestive heart failure as follows: (1) normal pulmonary blood flow with greater flow to the bases than apices; (2) equal pulmonary blood flow distribution to the upper and lower lobes; (3) upper lobe blood diversion; (4) alveolar airspace oedema. The cardiothoracic ratio on each film was independently measured by each radiologist. The diameter of the heart and the maximal transverse cardiac dimension were measured to the nearest $5 \mathrm{~mm}$. The average of the measurements of the two radiologists was calculated and used in statistical analyses.

\section{RADIONUCLIDE VENTRICULOGRAPHY}

Radionuclide ventriculography to determine the left ventricular ejection fraction was performed by standard gamma-camera computer gated techniques in supine patients. ${ }^{16-18}$ The analysis was conducted by a technologist blinded to the patient's clinical state. In our laboratory the intraobserver error of repeat computer analysis of the same ventriculographic data and the serial variability of studies performed on the same day in such patients was four ejection fraction units $(95 \%$ confidence interval) in patients with a left ventricular ejection fraction of $<0.40$. Others have shown that the interstudy variability of radionuclide ventriculography by this approach is independent of the day on which the study is performed. ${ }^{19}$ Differences in ejection fraction attributed to drug effect in a given patient were therefore assumed to be potentially important only if they were $>5$ ejection fraction units. All patients were studied before diltiazem treatment and 17 were also studied at least 72 hours after the start of treatment. To further assess the reproducibility of the results a third radionuclide ventriculographic study was performed either after discontinuation of diltiazem or when diltiazem treatment was reinitiated.

\section{EXPERIMENTAL PROTOCOL}

Twenty two patients were treated with oral diltiazem (mean $254 \mathrm{mg} / 24 \mathrm{~h}$ in four equal doses). The daily dose was increased by $120 \mathrm{mg} / 24 \mathrm{~h}$ until angina pectoris was adequately controlled or drug related side effects occurred. Diltiazem was stopped when increasing heart failure was accompanied by radiographic or radionuclide ventriculographic evidence of deteriorating left ventricular function. The distribution of doses was $120 \mathrm{mg}$ in one patient, $210 \mathrm{mg}$ in one patient, $240 \mathrm{mg}$ in 16 patients, $330 \mathrm{mg}$ in one patient, and $360 \mathrm{mg}$ in three patients. The patients were interviewed and examined carefully by one of us (MZ) at entry into study and every month afterwards.

Prescriptions were written for a five week supply of diltiazem tablets and were renewed only at the next follow up visit. From the date of the latest prescription issue, recorded by the pharmacist on the 
diltiazem bottle label, and by questioning the patient we assessed compliance. No new cardiac medications were given and the doses of patients' other cardiac medications were not altered. At each visit patients were asked whether their symptoms of heart failure had changed or become worse. In addition, we also asked whether they had been admitted to hospital for cardiac illness or respiratory insufficiency since the last follow up visit. Diltiazem was discontinued if side effects became intolerable or if the original indication for treatment was no longer present.

A posteroanterior chest radiograph was taken of the standing patient and a radionuclide left ventriculogram was obtained with the patient supine and at rest before diltiazem treatment was started. Repeat chest $x$ rays and radionuclide ventriculograms were performed in 19 and 17 patients a mean of 20 weeks (range 3-16 weeks) and 4 weeks (range 72 h-32 weeks), respectively after the start of diltiazem treatment. Supine bicycle exercise radionuclide ventriculography was not attempted in these patients, many of whom had previously demonstrated an extremely limited functional capacity during upright treadmill exercise testing.

\section{STATISTICAL ANALYSIS}

Analysis of variance was used to determine significance of the differences among various means (SD). ${ }^{20}$ The two tailed Students $t$ test was used to determine the significance of differences (mean (SD)) between two groups if the $F$ value was significant. The proportions in two independent samples were compared by $\chi^{2}$ analysis corrected for continuity. ${ }^{21}$ When the expected frequencies were $<5$ we used Fisher's exact probability test instead.

\section{Results}

The mean and median follow up periods for the entire group of patients were 7.5 months and 6.2 months respectively (range 2 weeks to 16 months). Six patients were treated for less than two monthswith diltiazem being intentionally discontinued in five. Reasons for early discontinuation in these individuals included asymptomatic pseudo-Mobitz Type 2 second degree atrioventricular block with narrow QRS complex, intractable nausea, subsequent myocardial infarction, subsequent coronary artery vein bypass surgery, and cost containment. In an additional patient, increasing fatigue, exertional dyspnoea, and paroxysmal nocturnal dyspnoea prompted the discontinuation of diltiazem after 5 weeks' treatment.

The dose of diltiazem taken by patients $(n=16)$ followed for more than eight weeks (253 (59) $\mathrm{mg} / 24$ h) did not differ from that taken by those $(n=6)$ who
Table Symptoms of congestive heart failure

\begin{tabular}{llll}
\hline Symptom & $\begin{array}{l}\text { Patients } \\
\text { developing new } \\
\text { symptom }\end{array}$ & $\begin{array}{l}\text { Patients with } \\
\text { remission of } \\
\text { symptom }\end{array}$ & $\begin{array}{l}\text { Patients with } \\
\text { continuation of } \\
\text { symptom }\end{array}$ \\
\hline $\begin{array}{c}\text { Orthopnoea } \\
\text { Paroxysmal } \\
\text { nocturnal } \\
\text { dyspnoea }\end{array}$ & 1 & 0 & 4 \\
$\begin{array}{c}\text { Dyspnoea on } \\
\text { exertion }\end{array}$ & 4 & 1 & 1 \\
\hline
\end{tabular}

developed intolerable side effects early (257 (42) $\mathrm{mg} / 24 \mathrm{~h}$ ). The dose of diltiazem was reduced in a further two patients because of pronounced sinus bradycardia with junctional escape rhythm in one and headache unresponsive to analgesics in another. The table shows the development and response of pre-existing symptoms of congestive heart failure in the study group.

There was a weight change of $>3 \mathrm{lb}(1.35 \mathrm{~kg})$ in nine patients on diltiazem therapy-five gained weight and four lost it. The mean (SD) ventricular ejection fraction at rest was similar off $(0.28(0.09)$ and on $(0.26(0.08))$ diltiazem treatment $(n=17)$ (fig 1). If the ejection fraction was measured some time

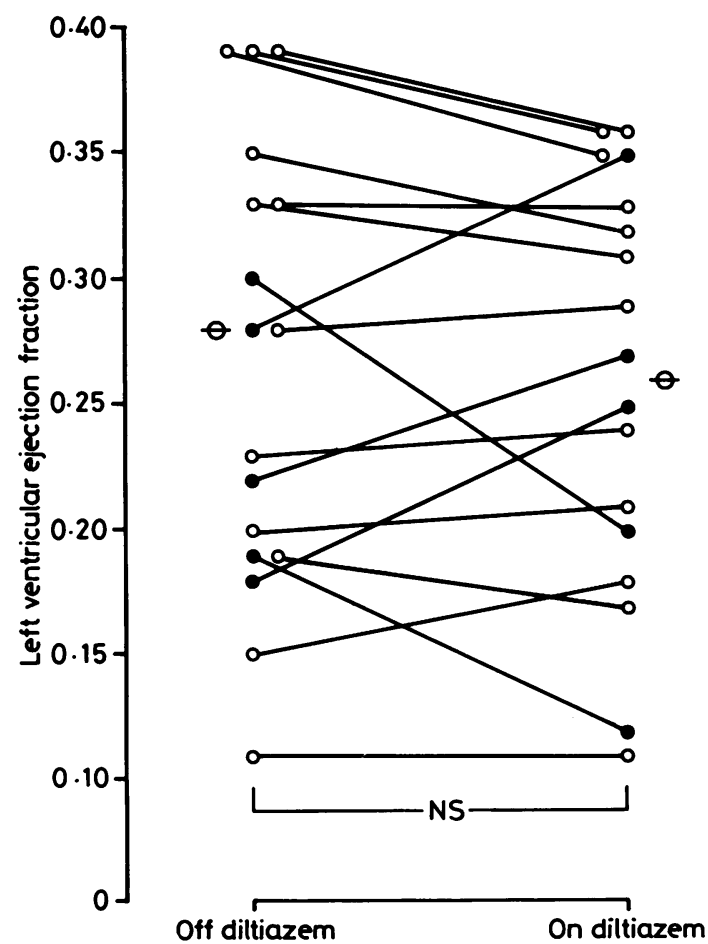

Fig 1 Left ventricular ejection fraction ( $L V E F$ ) at rest on oral diltiazem treatment and without diltiazem treatment. Mean dose of diltiazem $254 \mathrm{mg} / 24 \mathrm{~h}$. $\bigcirc$, patients with significant change in $L V E F ; O$, patients without significant change in LVEF; $\ominus$, mean LVEF. 


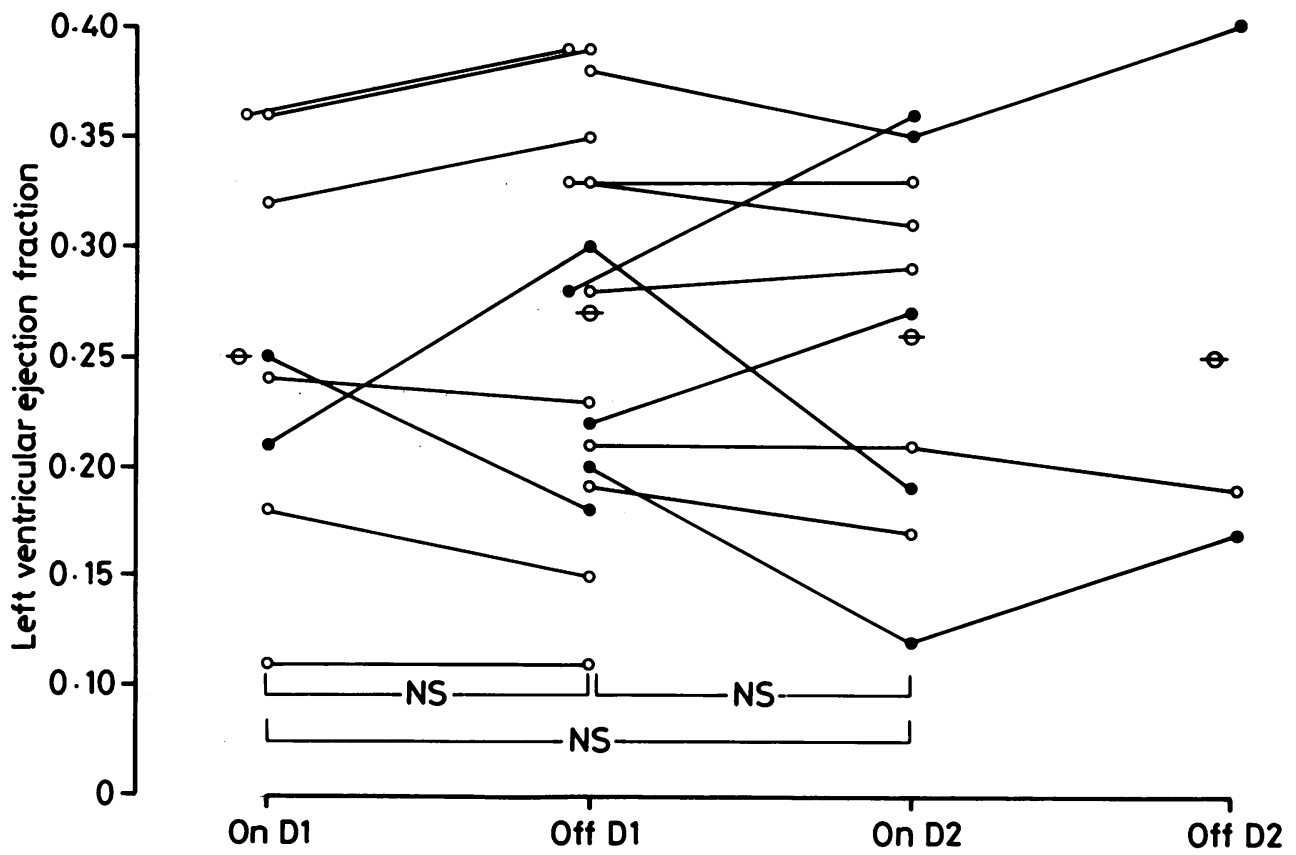

Fig 2 Left ventricular ejection fraction ( $L V E F)$ at rest off and on oral diltiazem in various patients at different times. "On D1" to "off D1" = 0.5-3.0 wk (mean = 1.3 (0.8) wk); "off D1" to "on D2" =0.5-76 $w k($ mean $=20.0(22 \cdot 1) w k)$; "on D2" to "off D2" = 2-40wk (mean = 14.7 (17.9) wk). 0 , patients with significant change in LVEF; $\bigcirc$, patients without significant change in LVEF; $\Theta$, mean LVEF. D, diltiazem.

later under identical clinical conditions, the results were averaged. Figure 2 shows the sequence of left ventricular ejection fraction determinations, with some patients having measurements performed off and on diltiazem on more than one occasion as mentioned above. There were significant changes in ejection fraction in five patients: in three there was an increase and in two a decrease. One of the two patients with a significant fall in ejection fraction had increasing fatigue and dyspnoea. The clinical condition of the other patient was unchanged.

The cardiothoracic ratio measured by chest $x$ ray was similar off $(0.47(0.06)$ and on $(0.48(0.05))$ diltiazem treatment ( $n=19 ; \mathrm{p}=\mathrm{NS}$ ) (fig 3 ). The cardiothoracic ratio changed by at least 0.03 in six patients-there was an increase in three and a decrease in three. Radiographic pulmonary blood flow score did not differ off $(1.14(0.6)$ and on $(1.7$ $(0.9))$ diltiazem $(\mathrm{n}=19)$. Pulmonary blood flow score changed by at least one unit in seven patients. It increased in five and decreased in two. One patient with increasing fatigue and dyspnoea had an increase in cardiothoracic ratio and pulmonary blood flow score and a significant decrease in left ventricular ejection fraction on diltiazem treatment.

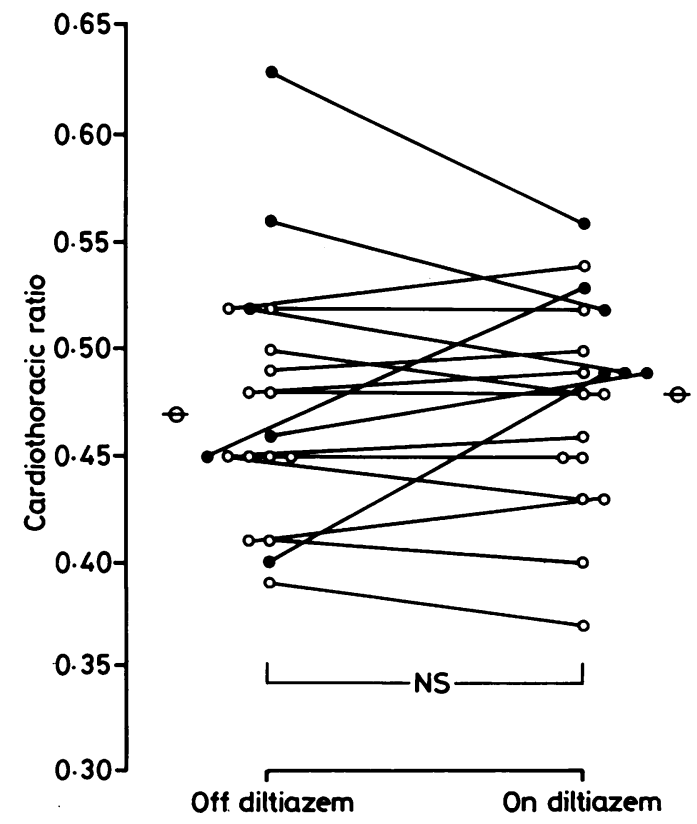

Fig 3 Cardiothoracic ratio (CTR) on posteroanterior chest radiograph off and on oral diltiazem. with change in $C T R$ of $\geqslant 0.03 ; 0$, patients with change in CTR of $<0.03 ; \ominus$, mean CTR. 


\section{Discussion}

Small scale studies have shown that intravenous diltiazem is well tolerated in patients with poor left ventricular function. Maurer et al reported radionuclide ventriculographic data on six patients with dilated cardiomyopathy given an intravenous bolus of diltiazem $(200 \mu \mathrm{g} / \mathrm{kg})$ followed by a continuous infusion $(20 \mu \mathrm{g} / \mathrm{kg} / \mathrm{min}) .{ }^{9}$ The left ventricular ejection fraction at rest was $0.32(0 \cdot 16)$ off and $0.35(0 \cdot 19)$ on diltiazem. Walsh et al used a similar intravenous protocol followed by a one day oral dose and reported the haemodynamic findings after invasive studies in eight patients with a mean baseline contrast ventriculographic ejection fraction of 0.24 (range $0 \cdot 11-0 \cdot 32) .{ }^{10}$ Intravenous diltiazem increased the mean stroke volume index of the group by $50 \%$, reduced systemic vascular resistance by $34 \%$, and reduced pulmonary artery wedge pressure by $34 \%$ without altering the maximal first derivative of left ventricular pressure $\left(\mathrm{dP} / \mathrm{dt}_{\max }\right)$. When oral diltiazem was given for 24 hours the haemodynamic effects were similar although pulmonary artery wedge pressure was not significantly reduced.

Neither of these studies, however, can answer the relevant clinical question: can oral diltiazem be used for longer term management of angina pectoris in patients with seriously compromised left ventricular function without precipitating congestive heart failure?

Our study was not a randomised controlled clinical trial; however, we did not regularly find deterioration of clinical, radiographic, or radionuclide descriptors of left ventricular function in our patients while they were on diltiazem treatment. Within five weeks of the start of oral diltiazem, however, at least one patient had an exacerbation of dyspnoea and fatigue accompanied by both a decrease in left ventricular ejection fraction and an increase in radiographically estimated cardiothoracic ratio and pulmonary venous pressure. The symptoms resolved and left ventricular ejection fraction returned to pre-treatment values within a week of discontinuation of diltiazem without any other intervention. Similarly, Walsh et al found a decrease in left ventricular $\mathrm{dP} / \mathrm{dt}_{\max }$ in two of six patients given intravenous diltiazem, although the mean value for their group of six subjects remained unchanged. ${ }^{10}$

Patients with ischaemic cardiomyopathy may make up a heterogenous group with a small percentage experiencing adverse clinical and haemodynamic effects when treated with diltiazem. Unfortunately, the limited number of patients in our study did not permit valid comparison of subgroups of patients on diltiazem to assess the effects of concomitant medications upon tolerance and safety in this clinical setting. In the present study the single patient in whom diltiazem was discontinued because of worsening left heart failure was also receiving maintenance treatment with digoxin, frusemide, and topical nitrates.

Our open label experience represents the largest published report to date on the use of diltiazem in the management of ischaemia in patients with severely compromised left ventricular function. Because it was a non-randomised, non-controlled trial, however, it cannot discriminate between drug-induced deterioration in left ventricular function and spontaneous deterioration caused by the patient's underlying structural heart disease. The results of the large, multicentre randomised, double blind, placebo controlled trial of diltiazem treatment after myocardial infarction that is now in progress should clarify this issue. It seems from the surprisingly benign course of disease in our patients treated for a median duration of 6.2 months with high dose oral diltiazem that this agent is not regularly associated with a deterioration in clinical, radiographic, or radionuclide ventriculographically assessed left heart performance-even when it is given long term to patients in whom such performance is already seriously compromised before treatment.

This work was made possible by a grant from Marion Laboratories Inc, Kansas City, Missouri, USA.

\section{References}

1 Fleckenstein A. Specific pharmacology of calcium in myocardium, cardiac pacemakers and vascular smooth muscle. Annu Rev Pharmacol Toxicol 1977;17:149-65.

2 Henry PD. Comparative pharmacology of calcium antagonists: nifedipine, verapamil, diltiazem. $\mathrm{Am} \mathrm{J}$ Cardiol 1980;46:1047-58.

3 Walsh RA, Badke RF, O'Rourke RA. Differential effects of systemic and intracoronary calcium channel blocking agents on global and regional left ventricular function in conscious dogs. Am Heart $J$ 1981;102:341-50.

4 Nakaya H, Schwartz A, Millard R. Reflex chronotropic and inotropic effects of calcium blocking agents in conscious dogs. Circ Res 1983;52:302-11.

5 Joshi PI, Dalal JJ, Ruttley MSJ, Sheridan DJ, Henderson AH. Nifedipine and left ventricular function in beta-blocked patients. $\mathrm{Br}$ Heart $J$ 1981;45:457-9.

6 Packer M, Miller J, Medina N. et al. Hemodynamic consequences of combined beta-adrenergic and slow calcium channel blockade in man. Circulation 1982;65:660-70.

7 Kieval J, Karaotein E, Kessler K, Mallon SM, Myerburg RJ. The effects of intravenous verapamil 
on hemodynamic status of patients with coronary artery disease receiving propranolol. Circulation 1982;65:653-8.

8 Ludbrook PA, Tiffenbrunn A, Reed FR, Sobel B. Acute hemodynamic responses to sublingual nifedipine: dependence on left ventricular function. Circulation 1982;65:489-98.

9 Maurer E, Nicoletti R, Brandt D, Klein W. Effect of calcium antagonists on cardiac performance in patients with dilated cardiomyopathy evaluated by non-invasive methods. Clin Cardiol 1983;6:399-404.

10 Walsh RW, Porter CB, Starling MR, O'Rourke RA. Beneficial effects of intravenous and oral diltiazem in severe congestive heart failure. $\mathrm{J} \mathrm{Am}$ Coll Cardiol 1984;3:1044-50.

11 Hurst JW, King SB III, Walter PF, Friesinger GC, Edwards JE. Atherosclerotic coronary heart disease: angina pectoris, myocardial infarction, and other manifestations of myocardial ischemia. In: Hurst JW, Logue RG, Rackley CE, et al, eds. The heart. New York: McGraw-Hill, 1982:1094.

12 Klein $\mathrm{HH}$, Schubothe $\mathrm{M}$, Nebendahl K, Kreuzer $\mathrm{H}$. The effect of two different diltiazem treatments on infarct size in ischemic reperfused porcine hearts. Circulation 1984;69:1000-5.

13 The Norwegian Multicentre Study Group. Timololinduced reduction in mortality and reinfarction in patients surviving acute myocardial infarction. $N$ Engl J Med 1981;304:801-7. 
14. Hjalmarson $\AA$, Elmfeldt D, Herlitz J, et al. Effect on mortality of metoprolol in acute myocardial infarction: a double-blind randomized study. Lancet 1981;ii:823-7.

15 Beta-blocker Heart Attack Trial Research Group. A randomized trial of propranolol in patients with acute myocardial infarction. JAMA 1982;247:1707-14.

16 Schelbert HR, Verga JW, Johnson AD, et al. Nontraumatic determination of left ventricular ejection fraction by radionuclide angiocardiography. Circulation 1975;51:902-9.

17 Marshall RC, Berger JH, Costin JC, et al. Assessment of cardiac performance with quantitative radionuclide angiocardiography. Circulation 1977;56: 820-9.

18 Maddox DE, Wyne J, Uren R, et al. Regional ejection fraction: a quantitative radionuclide index of regional left ventricular performance. Circulation 1979;59: 1001-9.

19 Wackers FT, Berger HJ, Johnstone DE, et al. Multiple gated cardiac blood pool imaging for left ventricular ejection fraction: validation of the technique and assessment of variability. Am J Cardiol 1979;43: 1159-66.

20 Runyon RP, Haber A. Fundamentals of behavioural statistics. Reading Massachusetts: Addison-Wesley, 1980:237.

21 Siegel S. Non-parametric statistics for the behavioral sciences. New York: McGraw-Hill, 1956:96. 